\title{
Влияние катионного и анионного замещений в дисульфиде и диселениде вольфрама на электропроводность и термоэдс
}

\author{
(C) Г.Е. Яковлева ${ }^{1}$, А.И. Романенко ${ }^{1}$, А.С. Бердинский ${ }^{2}$, В.А. Кузнецов ${ }^{1}$, \\ А.Ю. Леднева ${ }^{1}$, С.Б. Артемкина ${ }^{1}$, В.Е. Федоров ${ }^{1}$ \\ ${ }^{1}$ Институт неорганической химии Сибирского отделения Российской академии наук, \\ 630090 Новосибирск, Россия \\ ${ }^{2}$ Новосибирский государственный технический университет, \\ 630073 Новосибирск, Россия \\ E-mail: galina.yakovleva.91@mail.ru
}

(Получена 12 декабря 2016 г. Принята к печати 19 декабря 2016 г.)

\begin{abstract}
Исследованы температурные зависимости электропроводности и коэффициента термоэдс для серии образцов $\mathrm{W}_{1-x} \mathrm{Nb}_{x} \mathrm{~S}_{2}, \mathrm{~W}_{1-x} \mathrm{Nb}_{x} \mathrm{Se}_{2}, \mathrm{WS}_{2-y} \mathrm{Se}_{y}, \mathrm{~W}_{1-x} \mathrm{Nb}_{x} \mathrm{~S}_{2-y} \mathrm{Se}_{y}$ при низких температурах. Установлено, что катионное замещение $\mathrm{W}$ атомами $\mathrm{Nb}$ приводит к увеличению электропроводности и уменьшению коэффициента термоэдс. Анионное замещение S атомами Se приводит к одновременному увеличению электропроводности и коэффициента термоэдс. Наибольшее значение фактора мощности среди изученных образцов имеет материал $\mathrm{W}_{0.8} \mathrm{Nb}_{0.2} \mathrm{Se}_{2}$.
\end{abstract}

DOI: 10.21883/FTP.2017.06.44552.12

\section{1. Введение}

Термоэлектричество, основанное на эффекте Зеебека, вызывает особый интерес благодаря прямому преобразованию тепла в электричество. Более того, термоэлектрические генераторы могут использовать практически любые источники тепла, что существенно расширяет область их применения. Однако эффективность такого устройства ограничена эффективностью используемого термоэлектрического материала, которая характеризуется безразмерным параметром добротности $Z T=S^{2} \sigma T / \kappa$, где $S-$ коэффициент Зеебека, $\sigma-$ электропроводность, $\kappa$ - теплопроводность, $T-$ температура.

Дихалькогениды переходных металлов имеют слоистую структуру. Внутри слоя атомы связаны друг с другом прочными ковалентными связями, но между этими слоями имеют место только слабые ван-дер-ваальсовы взаимодействия [1]. Ряд работ посвящен изучению термоэлектрических свойств $\mathrm{WS}_{2}$ и $\mathrm{WSe}_{2}$ [2,3]. Данные материалы имеют высокий коэффициент Зеебека, низкую теплопроводность и низкую электропроводность. Поэтому целью нашей работы было увеличение электропроводности данных материалов и оценка влияния этого увеличения на фактор мощности.

Для увеличения электропроводности были синтезированы и исследованы образцы дихалькогенидов вольфрама с катионным и анионным замещением $\mathrm{W}$ атомами $\mathrm{Nb}$ и $\mathrm{S}$ атомами $\mathrm{Se}$.

\section{2. Методика эксперимента}

\section{1. Приготовление образцов}

Серии образцов $\mathrm{W}_{1-x} \mathrm{Nb}_{x} \mathrm{~S}_{2}, \mathrm{~W}_{1-x} \mathrm{Nb}_{x} \mathrm{Se}_{2}, \mathrm{WS}_{2-y} \mathrm{Se}_{y}$, $\mathrm{W}_{1-x} \mathrm{Nb}_{x} \mathrm{~S}_{2-y} \mathrm{Se}_{y}$ были синтезированы высокотемпера- турным ампульным методом. Для синтеза использовались элементы высокой степени чистоты. Исходные порошки металлов отжигали в потоке водорода при $1000^{\circ} \mathrm{C}$ в течение 1 ч, чтобы удалить адсорбированную воду и следы окислов. Стехиометрические количества металла и халькогена помещали в кварцевую ампулу. В дальнейшем ампулу вакуумировали и герметизировали. На последнем этапе ампулы дважды отжигались при температуре $T=850^{\circ} \mathrm{C}$ в течение 4 дней.

По данным рентгенофазового анализа образцы были однофазные, соответствующие типу $2 \mathrm{H}-\mathrm{WSe}_{2}$ и $2 \mathrm{H}-\mathrm{WS}_{2}$ соответственно.

\section{2. Методы измерения}

Для исследования температурных зависимостей синтезированные порошки были спрессованы в таблетки, из которых были вырезаны образцы размером $2 \times 2 \times 8$ мм. Контакты к образцам изготавливались с помощью графитовой пасты.

Температурные зависимости электропроводности были исследованы четырехконтактным методом в диапазоне температур $T=4.2-300 \mathrm{~K}$. Температурные зависимости коэффициента Зеебека были исследованы дифференциальным методом с переменным градиентом температуры в диапазоне температур $T=77-300 \mathrm{~K}$. Коэффициент Зеебека $S$ определялся согласно формуле

$$
S=\frac{U_{2}\left(\Delta T_{2}-U_{1}\left(\Delta T_{1}\right)\right)}{\Delta T_{2}-\Delta T_{1}},
$$

где $\Delta T_{1}, \Delta T_{2}$ - градиенты температуры, $U_{1}, U_{2}$ - термоэдс при соответствующих градиентах температуры. Все измерения проводились в гелиевой атмосфере. 

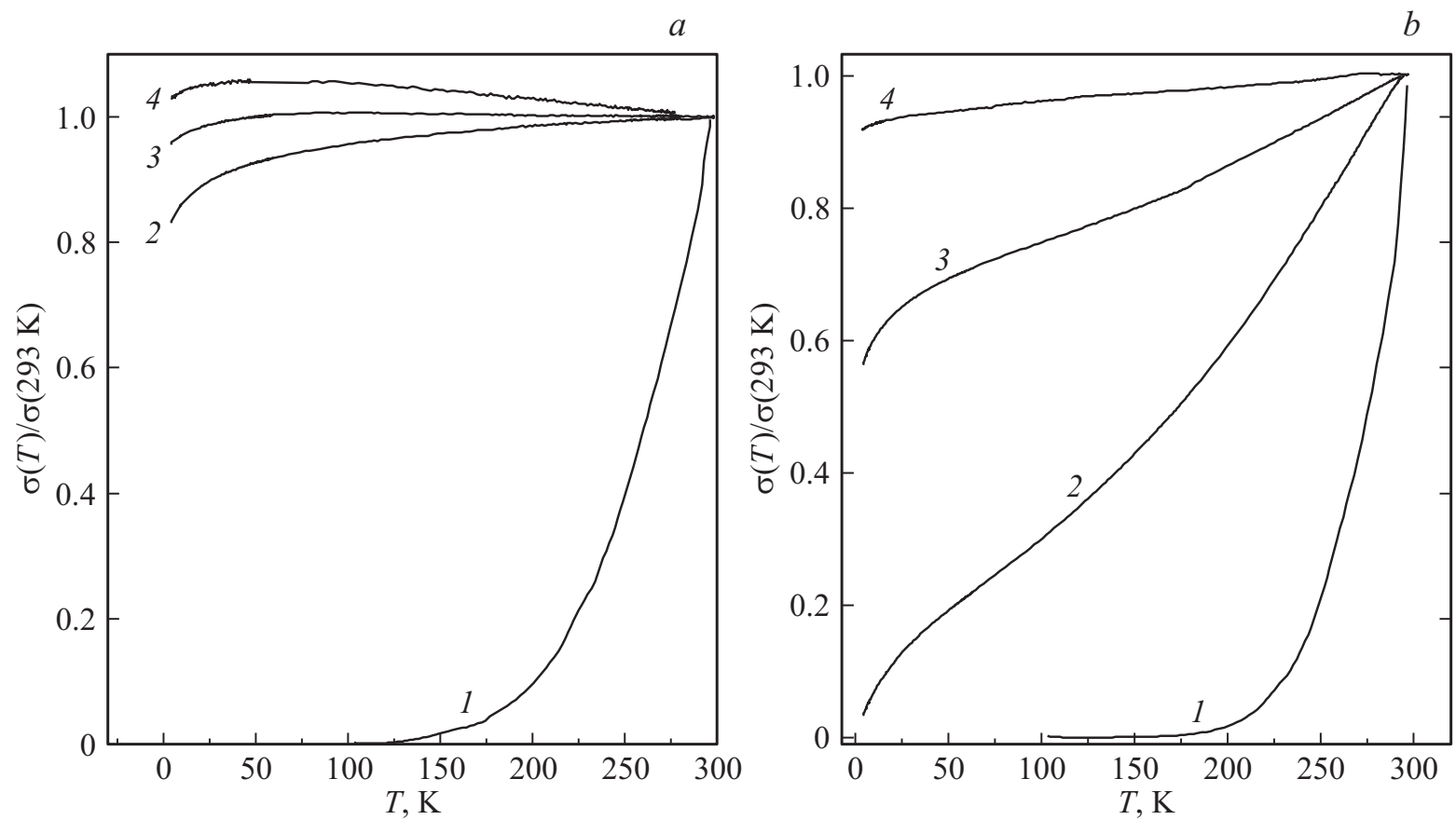

Рис. 1. Температурные зависимости электропроводности $\mathrm{W}_{1-x} \mathrm{Nb}_{x} \mathrm{Se}_{2}(a)$ и $\mathrm{W}_{1-x} \mathrm{Nb}_{x} \mathrm{~S}_{2}(b) . a, b-$ содержание $\mathrm{Nb} x, \%: 1-0$, $2-5,3-10,4-15$.
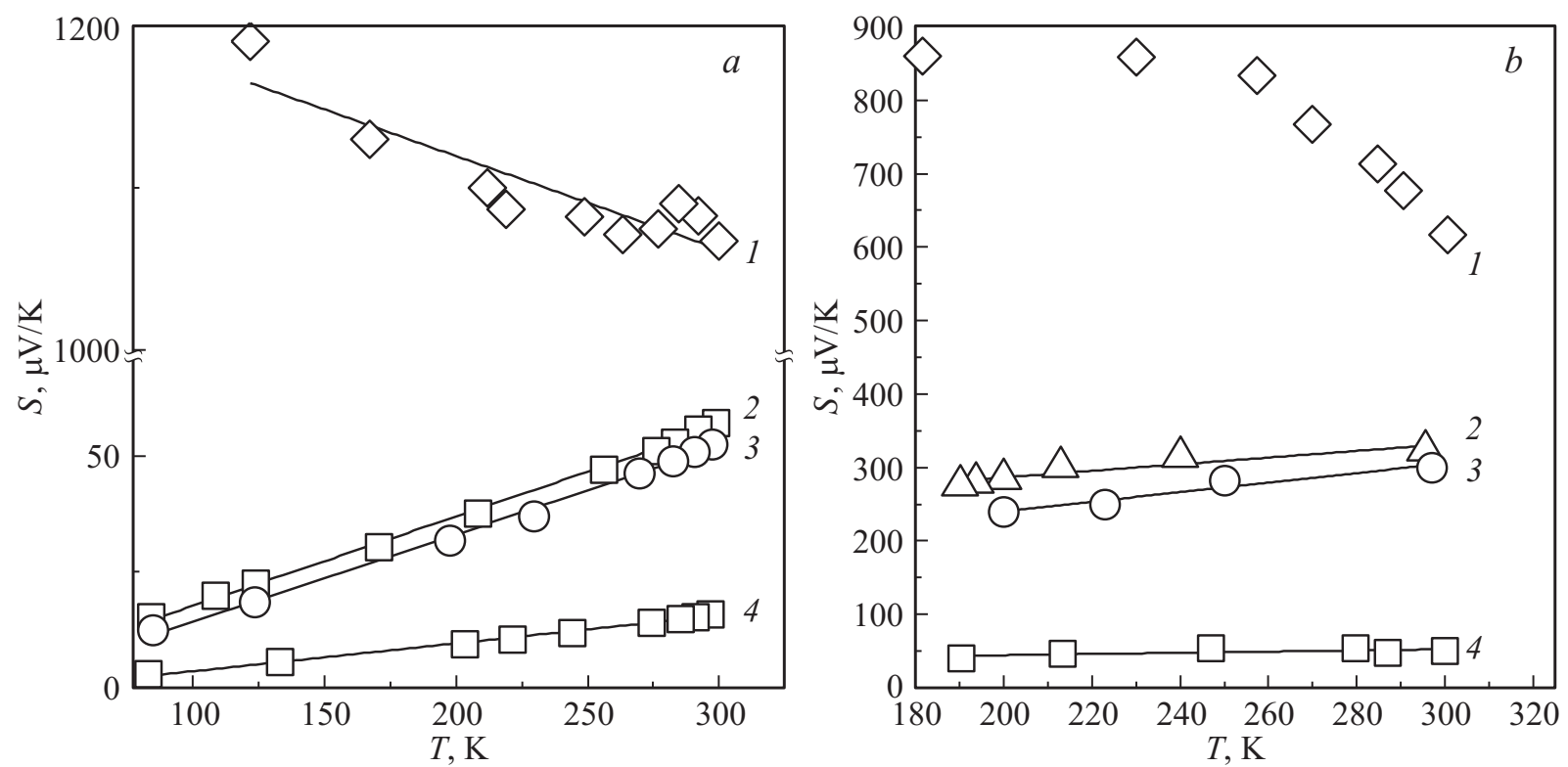

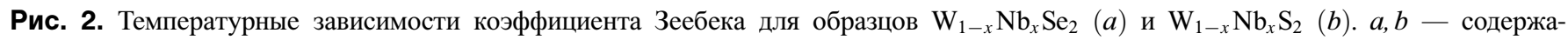
ние $\mathrm{Nb} x, \%: 1-0,2-5,3-10,4-15$.

\section{3. Экспериментальные данные}

\section{1. Исследование температурных зависимостей электропроводности}

Катионное замещение $W$ атомами $\mathrm{Nb}$ в дисульфиде и диселениде вольфрама приводит к увеличению электропроводности. Поскольку $\mathrm{WS}_{2}$ и $\mathrm{WSe}_{2}$ являются полупроводниками $p$-типа проводимости, добавление атомов $\mathrm{Nb}$, имеющих на внешней оболочке на один электрон меньше, приводит к образованию акцепторных примесей. В результате электропроводность материала увеличивается. Ранее это было показано на системе $\mathrm{Mo}_{1-x} \mathrm{Nb}_{x} \mathrm{~S}_{2}$ [4]. Температурные зависимости электропроводности $\mathrm{W}_{1-x} \mathrm{Nb}_{x} \mathrm{~S}_{2}$ и $\mathrm{W}_{1-x} \mathrm{Nb}_{x} \mathrm{Se}_{2}$ представлены на рис. 1.

Из рис. $1, a, b$ видно, что $\mathrm{WS}_{2}$ и $\mathrm{WSe}_{2}$ имеют полупроводниковый ход температурной зависимости элек- 
Таблица 1. Электропроводность $\mathrm{WS}_{2-y} \mathrm{Se}_{y}$ при $293 \mathrm{~K}$ в зависимости от содержания $\mathrm{Se}$

\begin{tabular}{c|c|c|c|c|c}
\hline$y(\mathrm{Se})$ & 0 & 0.1 & 0.2 & 0.25 & 2 \\
\hline$\sigma, \mathrm{CM} / \mathrm{M}$ & 0.04 & 0.12 & 0.46 & 0.61 & 2
\end{tabular}

Таблица 2. Фактор мощности исследуемых соединений при $293 \mathrm{~K}$

\begin{tabular}{l|c|c|c|c|c|c|c}
\hline \multirow{2}{*}{\multicolumn{1}{c|}{ Состав }} & \multicolumn{7}{c}{$x, y$} \\
\cline { 2 - 8 } & 0.01 & 0.02 & 0.05 & 0.10 & 0.15 & 0.20 & 0.25 \\
\hline $\mathrm{W}_{1-x} \mathrm{Nb}_{x} \mathrm{Se}_{2}$ & 270 & 277 & 72 & 40 & 6.5 & - & - \\
$\mathrm{W}_{1-x} \mathrm{Nb}_{x} \mathrm{~S}_{2}$ & - & - & 1.5 & 32 & 5 & - & - \\
$\mathrm{W}_{1-x} \mathrm{Nb}_{x} \mathrm{~S}_{0.1} \mathrm{Se}_{1.9}$ & 20 & 136 & - & - & - & - & - \\
$\mathrm{WS}_{2-y} \mathrm{Se}_{y}$ & - & - & - & 0.2 & - & 0.3 & 0.5
\end{tabular}

Примечание. Значения приведены в мкВт/м $\cdot \mathrm{K}^{2}$.

тропроводности. При этом добавление ниобия в $\mathrm{WSe}_{2}$ приводит к замене экспоненциальной температурной зависимости электропроводности на степенной зависимостью. При добавлении $15 \% \mathrm{Nb}$ происходит увеличение электропроводности на 4 порядка. Добавление $\mathrm{Nb}$ в $\mathrm{WS}_{2}$ также приводит к увеличению электропроводности.

В ряде работ $[5,6]$ показано, что анионное замещение атомов $\mathrm{S}$ атомами $\mathrm{Se}$ приводит к изменению запрещенной зоны материала. Было установлено, что добавление Se уменьшает величину запрещенной зоны. В свою очередь в полупроводниках уменьшение ширины запрещенной зоны приводит к увеличению электропроводности. Зависимости электропроводности при комнатной температуре от содержания $\mathrm{Se}$ в образце $\mathrm{WS}_{2-y} \mathrm{Se}_{y}$ представлены в табл. 1. Согласно работе [6], ширина запрещенной зоны для образцов имеет следующие значения: $\sim 1.81$ эВ в $\mathrm{WS}_{1.9} \mathrm{Se}_{0.1}, \sim 1.8$ эВ в $\mathrm{WS}_{1.8} \mathrm{Se}_{0.2}$, $\sim 1.79$ эВ в $\mathrm{WS}_{1.75} \mathrm{Se}_{0.25}$. Очевидно, что добавление $\mathrm{Se}$ ведет к значительно меньшему изменению электропроводности, чем при катионном замещении $\mathrm{W}$ атомами $\mathrm{Nb}$.

\section{2. Исследование температурных зависимостей коэффициента Зеебека}

Полупроводниковые материалы имеют обратно пропорциональную зависимость коэффициента Зеебека от температуры $(S \propto 1 / T)$. На рис. 2, $a$ представлены температурные зависимости коэффициента Зеебека для $\mathrm{W}_{1-x} \mathrm{Nb}_{x} \mathrm{Se}_{2}$. Видно, что добавление $\mathrm{Nb}$ уменьшает коэффициент Зеебека и меняет характер зависимости на металлический $(S \propto T)$. В случае дисульфида вольфрама добавление $\mathrm{Nb}$ также уменьшает коэффициент Зеебека.

Температурные зависимости для $\mathrm{W}_{1-x} \mathrm{Nb}_{x} \mathrm{~S}_{2}$ представлены на рис. $2, b$.

$\mathrm{B}$ образцах $\mathrm{WS}_{2-y} \mathrm{Se}_{y}$ анионное замещение $\mathrm{S}$ атомами Se привело к увеличению коэффициента Зеебека по сравнению с исходным дисульфидом вольфрама. Температурные зависимости коэффициента Зеебека представлены на рис. 3. Возможным объяснением такого эффекта является малая концентрация носителей.

Так как и катионное, и анионное замещения имеют разное влияние на термоэлектрические характеристики, были исследованы образцы $\mathrm{W}_{1-x} \mathrm{Nb}_{x} \mathrm{~S}_{2-y} \mathrm{Se}_{y}$. Результаты исследования представлены на рис. 4.

При добавлении 1 и $2 \% \mathrm{Nb}$ в $\mathrm{WSe}_{2}$ не изменяется вид температурной зависимости электропроводности по отношению к $\mathrm{WSe}_{2}(\sigma \propto T)$. Однако в образце

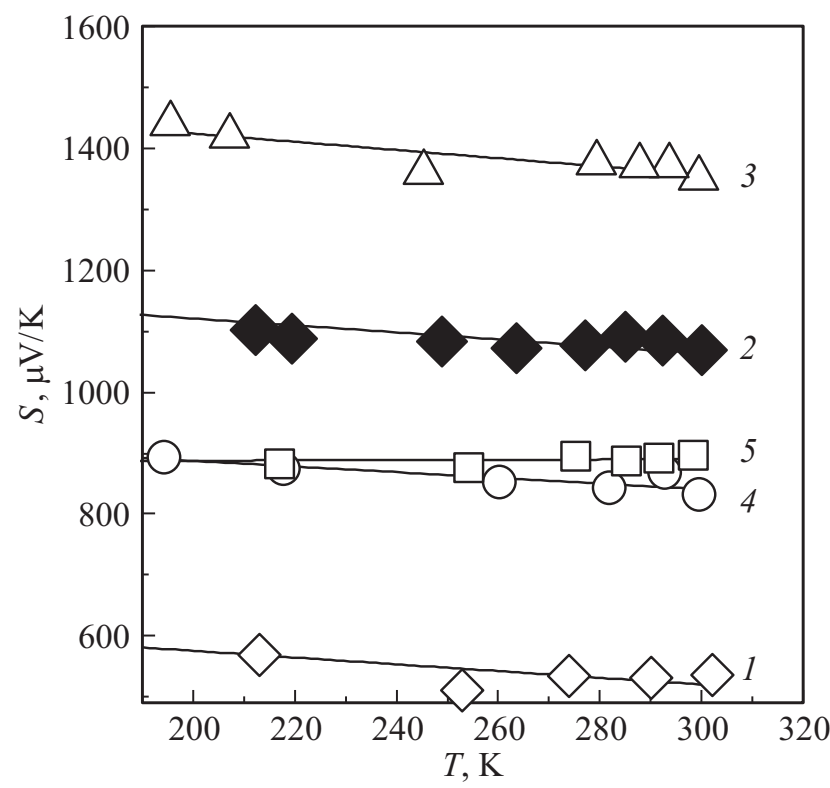

Рис. 3. Температурные зависимости коэффициента Зеебека для образцов $\mathrm{WS}_{2-y} \mathrm{Se}_{y}: 1-\mathrm{WS}_{2}, 2-\mathrm{WSe}_{2}, 3-\mathrm{WS}_{1.9} \mathrm{Se}_{0.1}$, $4-\mathrm{WS}_{1.8} \mathrm{Se}_{0.2}, 5-\mathrm{WS}_{1.75} \mathrm{Se}_{0.25}$.

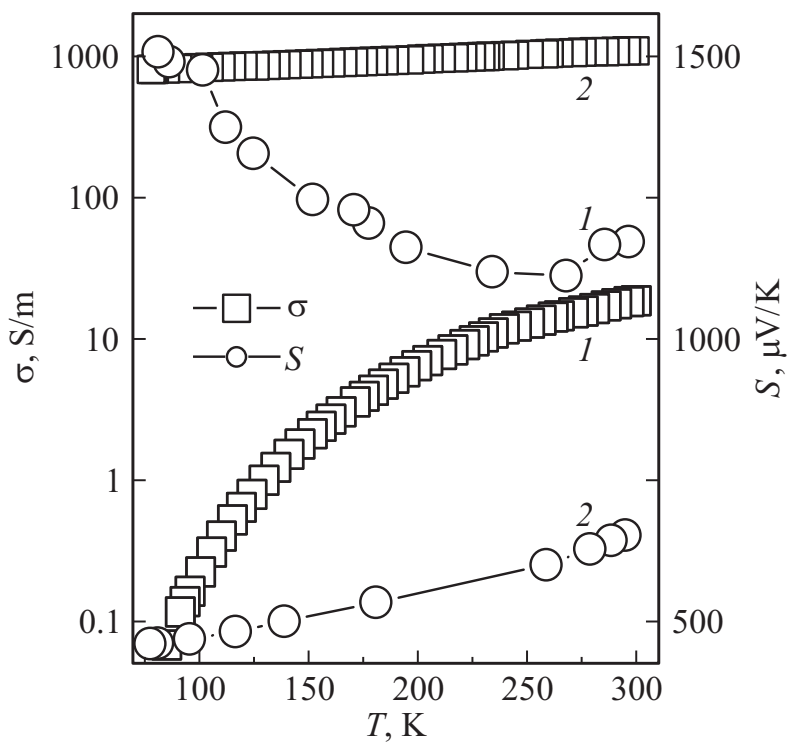

Рис. 4. Температурные зависимости электропроводности и коэффициента термоэдс: $1-\mathrm{W}_{0.99} \mathrm{Nb}_{0.01} \mathrm{~S}_{0.1} \mathrm{Se}_{1.9}, 2$ $\mathrm{W}_{0.98} \mathrm{Nb}_{0.02} \mathrm{~S}_{0.1} \mathrm{Se}_{1 . .9}$. 
$\mathrm{W}_{0.98} \mathrm{Nb}_{0.02} \mathrm{~S}_{0.1} \mathrm{Se}_{1.9}$ ход температурной зависимости меняется на металлический $(\sigma \propto 1 / T)$. При этом значения коэффициента термоэдс больше в образцах с двойным замещением. Для сравнительного анализа был рассчитан фактор мощности. Результаты представлены в табл. 2.

\section{4. Заключение}

В данной работе было изучено влияние катионного и анионного замещений в $\mathrm{W}_{1-x} \mathrm{Nb}_{x} \mathrm{~S}_{2}, \mathrm{~W}_{1-x} \mathrm{Nb}_{x} \mathrm{Se}_{2}$, $\mathrm{WS}_{2-y} \mathrm{Se}_{y}, \mathrm{~W}_{1-x} \mathrm{Nb}_{y} \mathrm{~S}_{2-y} \mathrm{Se}_{y}$ на термоэлектрические свойства при низких температурах. Наилучшее значение фактора мощности имеют материалы с катионным замещением атомов W атомами $\mathrm{Nb}$. Максимальное значение фактора мощности в образцах с двойным замещением примерно в 2 раза меньше фактора мощности лучших составов $\mathrm{W}_{1-x} \mathrm{Nb}_{x} \mathrm{Se}_{2}$. Однако при двойном замещении возможно более эффективное подавление решеточной теплопроводности, поэтому термоэлектрическая эффективность $Z T$ образцов $\mathrm{W}_{1-x} \mathrm{Nb}_{x} \mathrm{~S}_{2-y} \mathrm{Se}_{y}$ может оказаться выше, чем $\mathrm{WS}_{2-y} \mathrm{Se}_{y}$. Этот вопрос требует дальнейшего исследования.

Работа выполнена при поддержке гранта Российского научного фонда № 14-13-00674.

\section{Список литературы}

[1] H. Wang, H. Yuan, S.S. Hong, Y. Li, Y. Cui. Royal Soc. Chem., 44, 2664 (2015).

[2] J.Y. Kim, S.M. Choi, W.S. Seo, W.S. Cho. Korean Chem. Soc., 3, 3225 (2010).

[3] G.K. Solanki, D.N. Gujarathi, M.P. Lakshminarayana, M.K. Agarwal. Cryst. Res. Technol., 43, 179 (2008).

[4] V.E. Fedorov, N.G. Naumov, A.N. Lavrov, M.S. Tarasenko, S.B. Artemkina, A.I. Romanenko, M.V. Medvedev. Proc. 36th Int. Conf. on Information and Communication Technology, Electronics and Microelectronics (Opatija, Croatia, 2013) p. 11.

[5] D.N. Gujarathi, G.K. Solanski, M.P. Deshpande, M.K. Agarwal. Mater. Sci. Semicond. Processing, 8, 576 (2005).

[6] J. Kang, S. Tongay, J. Li, J. Wu. J. Appl. Phys., 113, 143703 (2013).

Редактор Л.В. Шаронова

\section{Effect of anionic and cationic substitution on conductivity and thermopower in tungsten disulfide and tungsten diselenide}

\author{
G.E. Yakovleva ${ }^{1}$, A.I. Romanenko ${ }^{1}$, A.S. Berdinsky ${ }^{2}$, \\ V.A. Kuznetsov' ${ }^{1}$, A.Yu. Ledneva' ${ }^{1}$, S.B. Artemkina ${ }^{1}$, \\ V.E. Fedorov ${ }^{1}$ \\ ${ }^{1}$ Nicolaev Institute of Inorganic Chemistry, \\ Russian Academy of Sciences, \\ 630090 Novosibirsk, Russia \\ 2 Novosibirsk State Technical University, \\ 630073 Novosibirsk, Russia
}

Abstract The temperature dependences of electrical conductivity and thermopower of $\mathrm{W}_{1-x} \mathrm{Nb}_{x} \mathrm{~S}_{2}, \mathrm{~W}_{1-x} \mathrm{Nb}_{x} \mathrm{Se}_{2}, \mathrm{WS}_{2-y} \mathrm{Se}_{y}$, $\mathrm{W}_{1-x} \mathrm{Nb}_{x} \mathrm{~S}_{2-y} \mathrm{Se}_{y}$ samples have been investigated in low temperature range. It is found that cationic substitution of $\mathrm{W}$ by $\mathrm{Nb}$ leads to increase of electrical conductivity and to decrease of thermopower. Anionic substitution of $\mathrm{S}$ by Se leads to increase of both, electrical conductivity and thermopower. $\mathrm{W}_{0.8} \mathrm{Nb}_{0.2} \mathrm{Se}_{2}$ has the best value of power factor among the samples prepared. 